\title{
First reported case of concurrent sonidegib and radiotherapy for recurrent, advanced basal cell carcinoma
}

\author{
Kaidi Wang, Mausam Patel, Arpan V. Prabhu, Gary D. Lewis \\ Department of Radiation Oncology, University of Arkansas for Medical Sciences, Little Rock, Arkansas, United States
}

\begin{abstract}
Basal cell carcinoma (BCC) is the most common human malignancy. Systemic therapy with a sonic hedgehog (SHH) pathway inhibitor plays an important role in the treatment of advanced BCC. Literature on concurrent use of radiation therapy (RT) with $\mathrm{SHH}$ inhibitors has been minimal and has solely been focused on vismodegib. We present a case report of a patient with recurrent basal cell carcinoma involving the high-risk area of the face, who was denied surgery due to comorbidities and difficulty in obtaining complete tumor removal without cosmetic or functional impairment. The patient received combined treatment of fractionated radiation with concurrent sonidegib and had complete clinical response with no significant toxicities. This is the first reported case on the use of concurrent RT with sonidegib for management of recurrent basal cell carcinoma of the head and neck.
\end{abstract}

Key words: basal cell carcinoma; radiation oncology; concurrent radiotherapy; systemic therapy; sonic hedgehog Rep Pract Oncol Radiother 2021;26(1):149-152

\section{Introduction}

Basal cell carcinoma (BCC) is the most common human malignancy, with an incidence rate of over 4 million cases annually. Standard surgical excision or Mohs micrographic surgery is the most effective and efficient means and the primary modality of treatment [1]. Primary RT is an option for patients with high-risk BCC who are poor surgical candidates and is an important option for cosmetic and functional preservation. In advanced BCC, however, which is defined as metastatic BCC (mBCC, a rare scenario for $B C C$ that refers to distant spread to another organ or nonregional lymph node or skin involvement) and locally advanced (laBCC) that are not amenable to curative surgery or RT [2], systemic therapy with a hedgehog pathway inhibitor should be considered per current guidelines from the American Society for Radiation Oncology
(ASTRO), National Comprehensive Cancer Network (NCCN) and American Academy of Dermatology (AAD) guidelines.

The two sonic hedgehog (SHH) pathway inhibitors approved by the U.S. Food and Drug administration for advanced BCC are vismodegib (Erivedge) and sonidegib (Odomzo). They work as cell surface receptor smoothened homolog (SMO) inhibitors, blocking activation of the hedgehog pathway [3]. Vismodegib has been used successfully and reported in several series of patients with advanced or metastatic BCC. Literature reports on concurrent use of RT with SMO inhibitors have been few and far between, and were mostly focused on vismodegib. Current ASTRO guidelines suggest avoiding concurrent use of radiation and SMO inhibitors due to the lack of clinical data demonstrating tolerability. Here, we report the first case of concurrent RT with sonidegib in a patient with

Address for correspondence: Kaidi Wang, M.D., Department of Radiation Oncology, University of Arkansas for Medical Sciences, 4301 W Markham Rd, Little Rock, AR 72205, tel: 501 526-8831; e-mail: kwang@uams.edu 
recurrent $\mathrm{BCC}$ of the head and neck who was not a surgical candidate.

\section{Case presentation}

The patient was an 85-year-old male with past medical history of coronary artery disease, myocardial infarction, stent placement and bypass graft surgery, hypertension, type 2 diabetes mellitus, and multiple non-melanoma cutaneous skin cancers elsewhere on the head and neck treated with local ablation/excision only by dermatology. He was referred to radiation oncology clinic for stage II BCC of left nasal tip s/p surgical resection and sonidegib.

His story began with a nasal tip skin lesion, for which he was followed by a dermatologist. Outside biopsy on 4/23/18 showed basal cell carcinoma, nodular type. It was treated several times by the dermatologist before he was referred to otolaryngology. He was seen by otolaryngology and noted to have a $3 \mathrm{~cm}$ lesion involving the left alar rim, nasal tip, and left nasal sidewall (Fig. 1A). Maxillofacial computed tomography (CT) scan showed a $1.6 \mathrm{~cm}$ lesion in the left side of the nose with surrounding skin thickening. There was underlying thinning of the left nasal bone, indeterminate for nasal bony invasion. Staging workup was consistent with T2N0M0, stage II, BCC. Given his cardiac and renal conditions and extensive disease prohibitive of surgical resection without cosmetic deformity, he was deemed unsuitable for surgery and referred to medical oncology.

He was seen by medical oncology and was started on sonidegib ( $200 \mathrm{mg}$ daily). He had an excellent initial response with $85-90 \%$ reduction in size clinically (Fig. 1B) and minimal side effects except for fatigue. Restaging imaging showed improved, but persistent residual disease. After being on sonidegib for 3 months, he was referred to Radiation Oncol- ogy for evaluation. Given the risk of developing resistance to $\mathrm{SHH}$ inhibitor sonidegib and need to treat his disease definitively, RT was recommended and patient agreed to proceed.

Patient received intensity-modulated radiation therapy with $70 \mathrm{~Gy}$ in 35 fractions with concurrent sonidegib. A $1 \mathrm{~cm}$ CTV margin and a $0.3 \mathrm{~mm}$ PTV margin were used. A thermoplastic mask was used for immobilization. Images from his first day and last day of radiation treatment are displayed in Figures $1 \mathrm{~B}$ and $1 \mathrm{C}$, respectively. He tolerated the treatment well and side effects were limited to fatigue, crusting inside his nasal passage, and erythema of his nasal skin managed with symptomatic treatment. On 1-month follow-up, patient reported resolving acute symptoms. He was noted to have a good response clinically on exam (Figure 1D). On 7-month follow-up, patient had finished sonidegib four months prior (total duration of 8 months) and had no residual disease clinically (Fig. 1E). Repeat CT imaging showed decreased thickening of soft tissue over the left nasal cartilage consistent with scar tissue without any focal lesion. The patient's CT imaging over time is displayed in Figure 2.

\section{Discussion}

Sonidegib has only been used as a single agent in advanced BCC. It was approved by FDA based on the phase II, multicenter, randomized and doubleblinded BOLT clinical trial [4]. It evaluated two doses of sonidegib (800 mg or $200 \mathrm{mg}$ daily) in patients with laBCC or mBCC until disease progression or unacceptable toxicity. The study demonstrated a clear role of SMO inhibitors in the treatment of advanced BCC, with higher objective response ( $36 \%$ vs. $34 \%)$, lower adverse events, longer duration of treatment, a lower discontinuation rate and a more favorable benefit-to-risk profile in

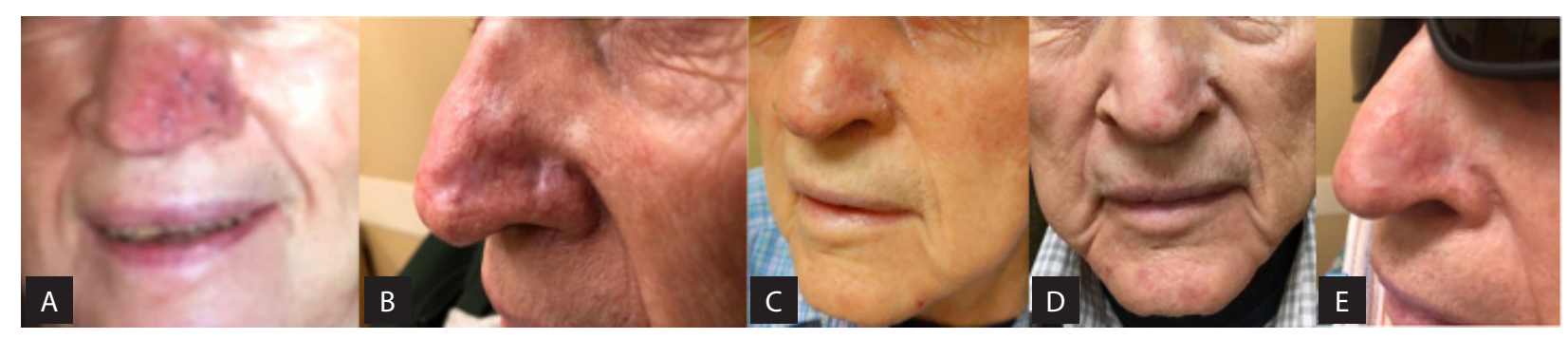

Figure 1. Clinical images at: A. Diagnosis; B. Initiation of radiotherapy (RT); C. Last day of RT; D. One month after RT; E. Seven month after RT 


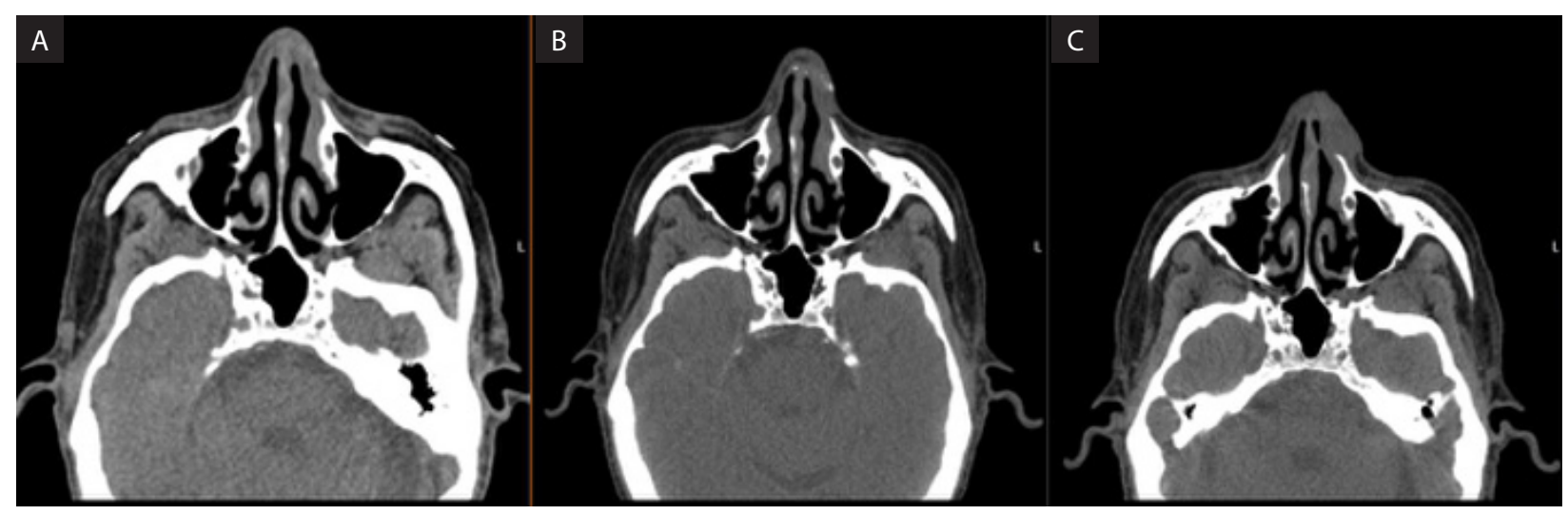

Figure 2. Computed tomography (CT) images from: A. 7-month follow-up visit; B. Before radiotherapy initiation after being on sonidegib for 3 month; $\mathbf{C}$. At the time of initial diagnosis. From right to left, resolution of original focal lesion can be clearly seen, as well as decreased thickening of the soft tissue over left nasal cartilage and minimal increased density of subcutaneous fat

$200 \mathrm{mg}$ dose compared to $800 \mathrm{mg}$ dose group. The most recent 42-month follow-up of BOLT study showed similar results and good safety and longterm efficacy [5]. Our patient, is the first reported case of the use of sonidegib concurrently with RT. The patient had excellent clinical response (Fig. 1D and $1 \mathrm{E}$ ) with minimal acute side effects.

There are several hypotheses on possible mechanisms of action for combination therapy with radiation and $\mathrm{SHH}$ inhibitors. Ionizing radiation works by causing direct and indirect damage to cellular DNA and the sonic hedgehog pathway has been found to be responsible for resistance to radiotherapy and chemotherapy and recurrence in multiple types of cancers [6]. Therefore, combining radiotherapy and a sonic hedgehog inhibitor makes sense theoretically. Molecular and animal studies so far indicate that X-rays induce expression of selected $\mathrm{SHH}$ target genes, and that $\mathrm{SHH}$ signaling is involved in the maintenance of cancer stem cells, which are inherently resistant to radiation and some chemotherapies [7, 8]. Gu et al. also demonstrated that the combination of focal radiation with SHH inhibitor has 'more than additive' inhibition of tumor metastasis as shown in an orthotopic mouse model for pancreatic cancer. There was also increased sensitivity to radiation in both the aforementioned mouse model and in basal cell carcinoma and head and neck squamous cell carcinoma cell lines $[8,9]$.

Vismodegib has been used previously concurrently with RT, albeit in small clinical series. Pollom et al. reported 2 cases of recurrent advanced
BCC treated with concurrent RT and vismodegib with good efficacy and tolerance, and no evidence of progressive disease at follow-up [10]. Another case reported by Raleigh et al. on auricular laBCC treated with induction vismodegib and radiation showed durable local control and acceptable level of acute toxicity [11]. Schulze et al. reported 4 cases (3 recurrent BCC, 1 with locoregional lymph node involvement) treated with concurrent vismodegib and RT. Three of the 4 patients experienced a persistent complete response, whereas one case remained stable for 6 months before further tumor progression [12].

There are no trials directly comparing sonidegib with vismodegib. Existing studies attempted to indirectly compare the BOLT (sonidegib) and ERIVANCE (vismodegib) trials using statistical adjustments. Odom et al. found that in laBCC, sonidegib has a higher objective response rate (ORR), longer median progression-free survival, and longer median duration of response [13]. Similarly, Dummer et al. found higher ORR for sonidegib, yet same efficacy and tolerability profile of the two drugs [14]. In mBCC however, sonidegib has a lower ORR compared to vismodegib [15]. In terms of their cost, the two drugs have comparable average wholesale price and cost per year, with sonidegib being slightly more affordable [16].

\section{Conclusion}

Our case report, to our knowledge, is the first reported case of concurrent use of RT and sonidegib 
in recurrent BCC. The patient had excellent clinical response with minimal acute side effects. Combination therapy of SHH inhibitors so far has only been limited to case reports, with no phase II trials reported. We have explored possible mechanisms regarding the synergistic efficacy of RT and $\mathrm{SHH}$ inhibitors as well as $\mathrm{SHH}$ inhibitors' potential for radiosensitization. Future directions include potential synergistic toxic effects and impaired wound healing due to SHH inhibitors [10]. Our report shows that concurrent sonidegib and RT is feasible and additional prospective study is needed to determine the optimal path to safely and effectively treat patients with laBCC.

\section{Conflict of interest}

All authors declare that conflicts of interest do not exist.

\section{Funding}

There was no funding obtained for this project.

\section{References}

1. Sumaira Z Aasi. Treatment and prognosis of basal cell carcinoma at low risk of recurrence. https://www.uptodate.com/ contents/treatment-and-prognosis-of-basal-cell-carcinoma-at-low-risk-of-recurrence\#H4133726805 (June, 2020).

2. Fecher LA, Sharfman WH. Advanced basal cell carcinoma, the hedgehog pathway, and treatment options - role of smoothened inhibitors. Biologics. 2015; 9: 129-140, doi: 10.2147/BTT.S54179, indexed in Pubmed: 26604681.

3. Jain S, Song R, Xie J. Sonidegib: mechanism of action, pharmacology, and clinical utility for advanced basal cell carcinomas. Onco Targets Ther. 2017; 10: 1645-1653, doi: 10.2147/OTT.S130910, indexed in Pubmed: 28352196.

4. Doan HQ, Silapunt S, Migden MR. Sonidegib, a novel smoothened inhibitor for the treatment of advanced basal cell carcinoma. Onco Targets Ther. 2016; 9: 5671-5678, doi: 10.2147/OTT.S108171, indexed in Pubmed: 27695345.

5. Villani A, Costa C, Fabbrocini G, et al. Sonidegib: Safety and Efficacy in Treatment of Advanced Basal Cell Carcinoma. Dermatol Ther (Heidelb). 2020; 10(3): 401-412, doi: 10.1007/s13555-020-00378-8, indexed in Pubmed: 32297221.

6. Jain S, Song R, Xie J. Sonidegib: mechanism of action, pharmacology, and clinical utility for advanced basal cell carcinomas. Onco Targets Ther. 2017; 10: 1645-1653, doi: 10.2147/OTT.S130910, indexed in Pubmed: 28352196.
7. Konings K, Vandevoorde C, Belmans N, et al. The Combination of Particle Irradiation With the Hedgehog Inhibitor GANT61 Differently Modulates the Radiosensitivity and Migration of Cancer Cells Compared to X-Ray Irradiation. Front Oncol. 2019; 9: 391, doi: 10.3389/fonc.2019.00391, indexed in Pubmed: 31139573.

8. Gu D, Liu H, Su GH, et al. Combining hedgehog signaling inhibition with focal irradiation on reduction of pancreatic cancer metastasis. Mol Cancer Ther. 2013; 12(6): 10381048, doi: 10.1158/1535-7163.MCT-12-1030, indexed in Pubmed: 23468532.

9. Hehlgans S, Booms P, Güllülü Ö, et al. Radiation Sensitization of Basal Cell and Head and Neck Squamous Cell Carcinoma by the Hedgehog Pathway Inhibitor Vismodegib. Int J Mol Sci. 2018; 19(9), doi: 10.3390/ijms19092485, indexed in Pubmed: 30142876.

10. Pollom EL, Bui TT, Chang AL, et al. Concurrent Vismodegib and Radiotherapy for Recurrent, Advanced Basal Cell Carcinoma. JAMA Dermatol. 2015; 151(9): 998-1001, doi: $10.1001 /$ jamadermatol.2015.0326, indexed in Pubmed: 25874733.

11. Raleigh DR, Algazi A, Arron ST, et al. Induction Hedgehog pathway inhibition followed by combined-modality radiotherapy for basal cell carcinoma. Br J Dermatol. 2015; 173(2): 544-546, doi: 10.1111/bjd.13748, indexed in Pubmed: 25702621.

12. Schulze B, Meissner M, Ghanaati S, et al. Hedgehog pathway inhibitor in combination with radiation therapy for basal cell carcinomas of the head and neck : First clinical experience with vismodegib for locally advanced disease. Strahlenther Onkol. 2016; 192(1): 25-31, doi: 10.1007/s00066-015-0902-7, indexed in Pubmed: 26449347.

13. Odom D, Mladsi D, Purser M, et al. A Matching-Adjusted Indirect Comparison of Sonidegib and Vismodegib in Advanced Basal Cell Carcinoma. J Skin Cancer. 2017; 2017: 6121760, doi: $10.1155 / 2017 / 6121760$, indexed in Pubmed: 28607774.

14. Dummer R, Ascierto PA, Basset-Seguin N, et al. Sonidegib and vismodegib in the treatment of patients with locally advanced basal cell carcinoma: a joint expert opinion. J Eur Acad Dermatol Venereol. 2020; 34(9): 1944-1956, doi: 10.1111/jdv.16230, indexed in Pubmed: 31990414.

15. Sekulic A, Migden MR, Basset-Seguin N, et al. ERIVANCE BCC Investigators, ERIVANCE BCC Investigators. Long-term safety and efficacy of vismodegib in patients with advanced basal cell carcinoma: final update of the pivotal ERIVANCE BCC study. BMC Cancer. 2017; 17(1): 332, doi: 10.1186/s12885-017-3286-5, indexed in Pubmed: 28511673.

16. Kish T, Corry L. Sonidegib (Odomzo) for the Systemic Treatment of Adults With Recurrent, Locally Advanced Basal Cell Skin Cancer. P T. 2016; 41(5): 322-325, indexed in Pubmed: 27162473. 